\title{
Age differences in ocular demodicosis: Demodex profiles and clinical manifestations
}

\author{
Jing Li", Xiaohui Luo", Yinglin Liao, Lingyi Liang \\ State Key Laboratory of Ophthalmology, Zhongshan Ophthalmic Center, Sun Yat-sen University, Guangzhou, China \\ Contributions: (I) Conception and design: L Liang; (II) Administrative support: L Liang; (III) Provision of study materials or patients: L Liang; (IV) \\ Collection and assembly of data: X Luo, J Li; (V) Data analysis and interpretation: J Li, X Luo, Y Liao; (VI) Manuscript writing: All authors; (VII) \\ Final approval of manuscript: All authors. \\ "These authors contributed equally to this work. \\ Correspondence to: Lingyi Liang, MD, PhD. Zhongshan Ophthalmic Center, Sun Yat-sen University, 7 Jinsui Road, Guangzhou 510000, China. \\ Email: lianglingyi@gzzoc.com.
}

\begin{abstract}
Background: Demodex infestation is highly age-dependent. Intriguingly, our previous studies that focused on children and young adult patients suggested that the clinical features of young patients were different from those studies enrolling mainly elderly patients. Whether age plays a role between young and elderly patients with ocular demodicosis remains unclear.

Methods: This prospective comparative study included 91 patients younger than 35 years and 92 older than 45 years with ocular demodicosis. Demodex mite count, symptoms, tear film, and ocular changes were compared between the two groups. Risk factors of meibomian gland loss (MGL) and corneal changes were analysed in the two groups.
\end{abstract}

Results: Demodex counts were comparable between the two groups. Young patients had higher D. brevis counts and overall percentage of $D$. brevis, while elderly patients had more $D$. folliculorum (all $\mathrm{P}<0.05$ ). Irritation and blurred vision were more common in young patients, while eye fatigue and photophobia were more common in elderly patients (both $\mathrm{P}<0.05$ ). The two groups had comparable tear volume and tear break-up time. Meibomian gland dysfunction was the most common sign in both groups but MGL was significantly more severe in young patients. More prevalent corneal changes and more eyelash disorders were found in young patients (both $\mathrm{P}<0.05$ ). Female sex, a higher $D$. brevis percentage, lid margin anomalies, and MGL were associated with corneal change, while a higher D. brevis percentage and lid margin anomalies were related to MGL in young patients. MGL was associated with corneal change, but age was the only predictor of MGL in the elderly group.

Conclusions: Young patients with ocular demodicosis tend to have more D. brevis infestation, more MGL, and more corneal involvement.

Keywords: Demodex mite; meibomian gland; age

Submitted Nov 29, 2020. Accepted for publication Feb 16, 2021.

doi: $10.21037 / \mathrm{atm}-20-7715$

View this article at: http://dx.doi.org/10.21037/atm-20-7715

\section{Introduction}

Demodex folliculorum (D. folliculorum) and Demodex brevis (D. brevis) are the only two species of mites that affect humans $(1,2)$. D. folliculorum infests the hair follicle, while $D$. brevis resides in the sebaceous and meibomian glands (2-4). Ocular demodicosis has been implicated in ocular surface inflammation, including blepharitis, eyelash disorders, meibomian gland dysfunction (MGD), chalazia, and blepharoconjunctivitis (BKC) (5-10). The incidence of Demodex infestation increases with age: it is observed in $84 \%$ of the general population aged over 60 years and 
$100 \%$ of the general population over 70 years old but is reported to be very rare in healthy children under 16 years of age (11-13). However, we previously reported ocular Demodex infestation in 12 paediatric patients who were diagnosed with BKC but failed to respond to conventional treatments (8). To the best of our knowledge, this was the first time that Demodex infestation was detected in children without systemically immunocompromised status. Recently, we conducted an epidemiological survey and revealed a low Demodex mite count in $12.0 \%$ of healthy Chinese children aged between 3 and 14 years (14).

To mitigate the concern that the demodex infestation is age-related and much more prevalent in the elders, our previous studies focussed on young patients and found a high prevalence of keratitis $(14,15)$. Ahmad also reported 6 cases of keratopathy in patients with Demodex infestation, 2 of whom were under 35 years (9). We wondered whether young patients are more susceptible to corneal involvement than elderly patients. However, other studies that mostly included elderly patients have shown that the most common clinical features are MGD and eye dryness with predominantly $D$. folliculorum (15-20).

Whether age plays a role between young and elderly patients with ocular demodicosis remains unclear. Herein, for the first time, we conducted a prospective observational comparative study to investigate the differences in clinical features between young and elderly patients with ocular demodicosis.

We present the following article in accordance with the STROBE reporting checklist (available at http://dx.doi. org/10.21037/atm-20-7715).

\section{Methods}

This study followed the principles of the Declaration of Helsinki (as revised in 2013) for the protection of human subjects in medical research and was approved by the Ethics Committee of Zhongshan Ophthalmic Center, Sun Yat-sen University (No. 2017KYPJ042). All participants were given a full explanation of the study, and written informed consent was obtained.

\section{Patients}

A total of 183 patients with a confirmed diagnosis of ocular demodicosis were enrolled at the cornea outpatient department of Zhongshan Ophthalmic Center between March 2018 and July 2019. Patients were divided into two groups according to age: patients aged $\leq 35$ years were included in the young group, and patients aged $\geq 45$ years were included in the older group. All subjects were Chinese. Both eyes underwent examination, and the worse eye with more severe corneal fluorescein staining or more severe conjunctival injection was selected for comparison. If severity was equal in both eyes, the right eye was designated as the study eye. Patient with ocular diseases other than ocular demodicosis and senile cataract, prior ocular surgery history, and contact-lens wearers were excluded in our study. Any systemic disease was also excluded.

\section{Diagnosis of ocular demodicosis}

Symptomatic patents with ocular surface discomfort and clinical signs of cylindrical dandruff (CD) at eyelash roots were subjected to lash sampling and microscopic counting of Demodex as previously described $(8,16,17)$ by a masked technician who had no idea of patients' clinical information. In brief, two lashes were epilated from each eyelid under a slit-lamp microscope and placed on a glass slide. One drop of saline or fluorescein solution was applied to dissolve the CD and to allow embedded Demodex to migrate out (17). Demodex species and counts for each patient were recorded and whose total counts greater than or equal to 3 in 8 lashes were recorded as Demodex-positive (21). Those symptomatic patients with positive demodex count were diagnosed as ocular demodicosis $(20,21)$.

\section{Evaluation of ocular surface parameters}

The ocular surface examinations were as follows. Eyelid margin abnormalities were determined according to previously reported scoring criteria $(22,23)$. The shape of the palpebral margin was observed under a slit lamp and checked for congestion of the palpebral margin, blockage of the meibomian gland opening, irregular shape of the palpebral margin, and backward displacement of the meibomian gland orifice opening. The score was 0 for normal and 1 point for the presence of any one of the above findings. The accumulated scores were recorded as the palpebral margin morphology score (range, 0-4) $(22,23)$. Eyelash disorders were defined as trichiasis, $\mathrm{CD}$, or scaly discharge at the roots of lashes.

Tear break-up time (TBUT) was measured using fluorescein solution under slit-lamp (24). The severity of MGD was graded by meibography using a Keratograph 5 M (Oculus; Wetzlar, Germany) as previously reported; 
that is, 0 for no meibomian gland loss (MGL), 1-2 for less than one-third total MGL and considered as 'mild', 3-4 for one-third to two-thirds total MGL and considered as 'moderate', and 5-6 for more than two-thirds total MGL and considered as 'severe' (25). Tear meniscus height (TMH) was also evaluated under a Keratograph $5 \mathrm{M}$. According to a published corneal grading scale, corneal pathologies were graded by slit-lamp photographs as ' 0 ' for no abnormal finding, ' 1 ' for superficial punctate keratopathy (SPK) only or limbitis only, or ' 2 ' for stromal involvement such as infiltration or ulceration (7).

\section{Statistical analysis}

All statistical analyses were performed using SPSS version 22.0 (IBM, Armonk, NY). Two investigators performed grading of MGD and corneal changes. Any inconsistent grading between the two was arbitrated by a third investigator who did not know the other clinical information. Continuous variables are reported as means \pm standard deviation. Categorical variables were recorded as presence (yes) or absence (no) and reported as the number (percentage) of subjects. Variables were compared between groups using Student's $t$-test, the Kruskal-Wallis test, Pearson's $\chi^{2}$ test, and Fisher's exact test, as appropriate. Those with probable statistical significance $(\mathrm{P}<0.15)$ were subjected to multiple logistic regression to determine factors associated with corneal changes and MGL. In all analyses, $\mathrm{P}<0.05$ was considered statistically significant.

\section{Results}

\section{Demographic data}

Among 183 patients (73 males, 110 females) with ocular demodicosis, there were 91 patients $(17.7 \pm 6.9$ years, range: 6 to 30 years, 40 males and 51 females) in the young group and 92 patients $(55.5 \pm 9.3$ years, range: 45 to 81 years, 33 males and 59 females) in the elderly group. Sex was matched between the two groups $(\mathrm{P}=0.293$, Table 1). Details of patient demographics and clinical data are provided in Table 1.

\section{Prevalence of Demodex infestation and Demodex mite profiles}

The total Demodex count was comparable between young and elder patients $[6(4,8)$ vs. $7(4,13), \mathrm{P}=0.176]$. However, the $D$. brevis counts and the percentage of D. brevis to total Demodex (D. brevis \%) of the young group was significantly higher than that of the elderly group $[2(1,3)$ vs. $0(0,2)$, $43 \%(25 \%, 100 \%)$ vs. $4 \%(0,21 \%)$, both $\mathrm{P}<0.001$, Table 1$]$. In contrast, D. folliculorum was more dominant in the older group $[6(3,10)$ vs. $3(2,6), \mathrm{P}<0.001,96 \%(73 \%, 100 \%)$ vs. $57 \%(40 \%, 82 \%)$, both $\mathrm{P}<0.001$, Table 1]. Mixed infestation, i.e., both $D$. folliculorum and $D$. brevis were detected in $48.9 \%(45 / 92)$ and $78.0 \%$ (71/91) of elder and younger groups respectively.

\section{Symptom profiles}

Eye dryness, itching, fatigue, pain, blurred vision, photophobia, and redness were complaints common to all patients. Among these symptoms, itching and eye dryness were the two most common in both groups. Young patients had more blurred vision ( $40.7 \%$ vs. $9.8 \%, \mathrm{P}<0.001)$, more redness $(36.3 \%$ vs. $21.7 \%, \mathrm{P}=0.030)$, and more eye pain $(24.2 \%$ vs. $2.2 \%, \mathrm{P}<0.001)$, while older patients had more eye fatigue $(67.4 \%$ vs. $13.2 \%, \mathrm{P}<0.001)$ and more photophobia (54.3\% vs. $30.8 \%, \mathrm{P}=0.002)$.

\section{Ocular surface parameters}

Lid margin abnormalities were comparable between the two groups [2 (1, 3) vs. 2 (2, 3), $\mathrm{P}=0.998]$. More severe MGL was found in the young group (score $\geq 5,34 / 91,37.4 \% v s$. 14/92, 15.2\%, $\mathrm{P}<0.001$, Table 1).

MGD, blepharitis, and corneal change were the most common pathologies in the young group, while MGD and eye dryness were more common in the elderly group. TBUT and TMH were similar between groups (both $\mathrm{P}>0.05)$. The incidence of chalazia was higher in the young group than in the older group [12.1\% (11/91) vs. $2.2 \%$ (2/92), $\mathrm{P}=0.009]$.

\section{Prevalence and potential risk factors of corneal changes}

Corneal changes were detected in 67 out of 91 (73.7\%) patients in the young group, which was significantly higher than that in the elderly group $(36 / 92,39.1 \%, \mathrm{P}<0.001$, Table 1). Corneal findings included SPK or limbitis in $31.9 \%$ of patients and corneal stromal infiltration in $41.8 \%$ of young patients. In contrast, in the elderly group, SPK was the only corneal pathology in the 36 cases (39.1\%) with corneal complications (Table 1). None of the patients developed stromal infiltration or ulceration. 
Table 1 Demographics and clinical manifestations in young and elderly patients with ocular demodicosis

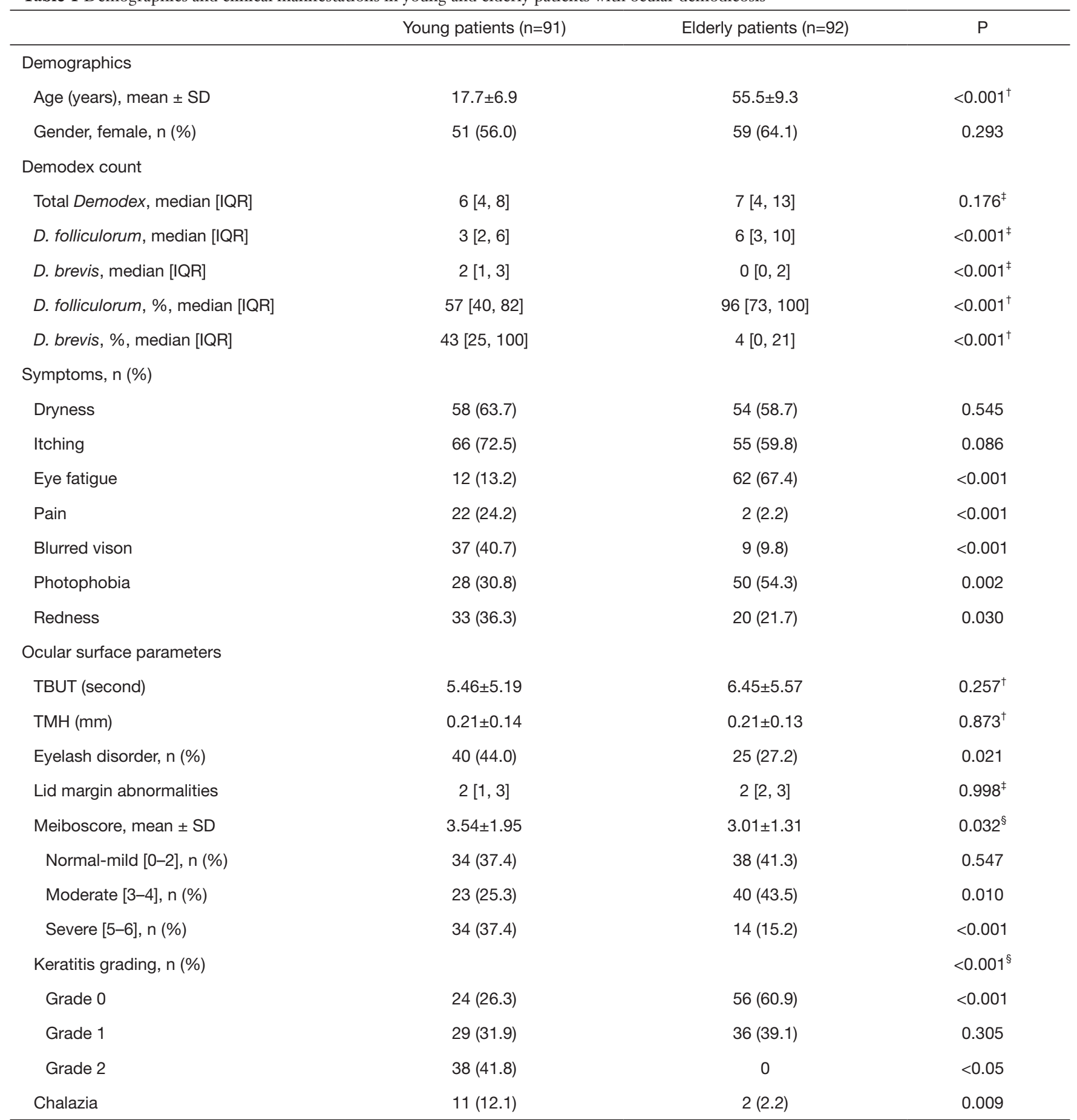

${ }^{\dagger}$, Student $t$ test; ${ }^{\ddagger}$, Kruskal-Wallis Test; ${ }^{\S}$, Fisher exact test. Pearson chi-squared test was used was used in the table except additional mentioned. TBUT, tear break-up time; TMH, tear meniscus height; $D$. folliculorum, Demodex folliculorum, Demodex brevis, $D$. brevis; $D$. folliculorum \%, D. folliculorum/total Demodex; D. brevis \%, D. brevis/total Demodex. 
Table 2 Probable risk factors of corneal changes in young and elderly patients

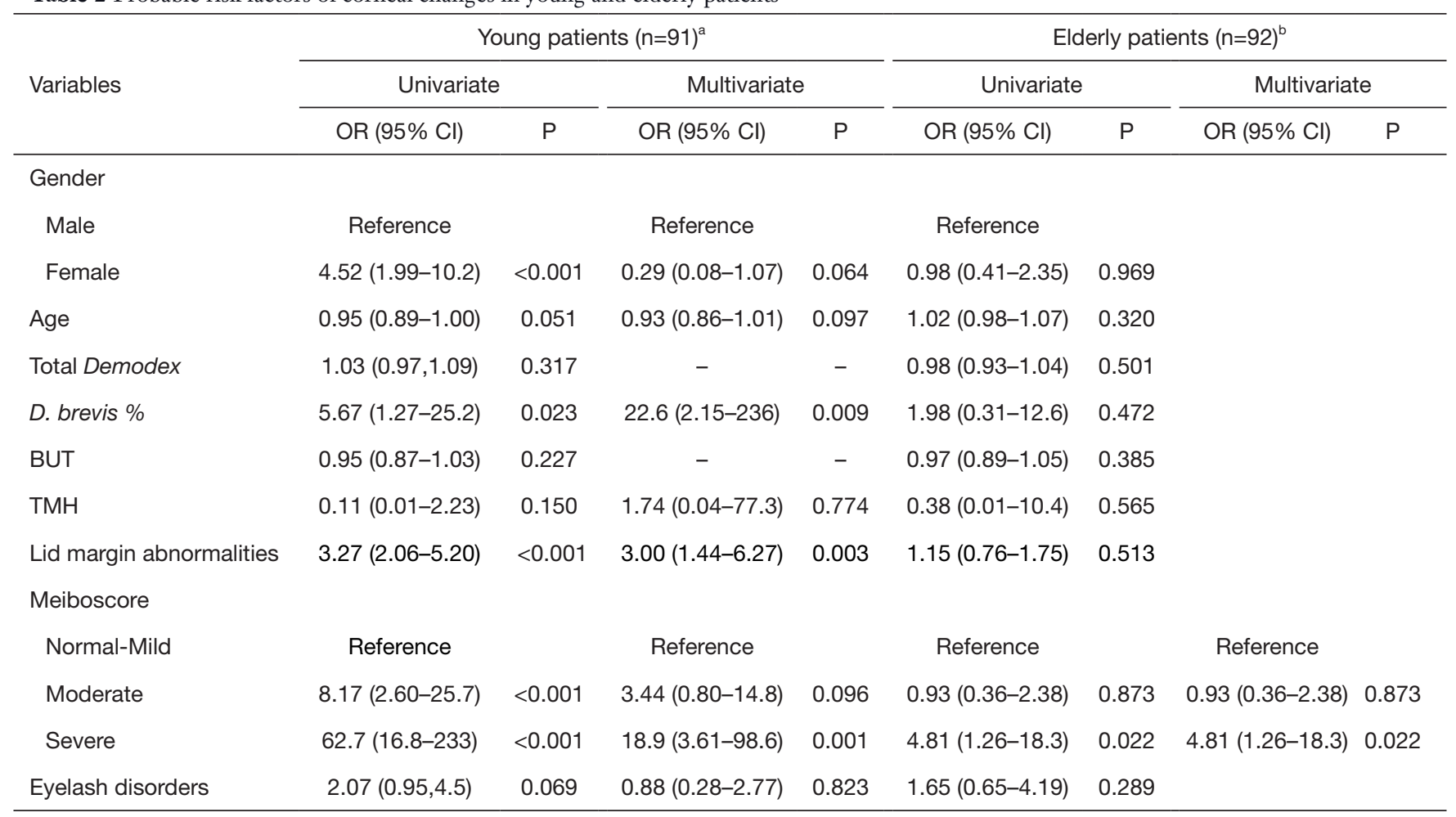

Variables which $\mathrm{P}<0.15$ were included in the multivariate ordinal logistic regression analysis. ${ }^{\mathrm{a}}$, Ordinal logistic regression; ${ }^{\mathrm{b}}$, binary logistic regression. $\mathrm{Cl}$, confidence interval; OR, odds ratio; BUT, tear break-up time; TMH, tear meniscus height. D. brevis, Demodex brevis; $D$. brevis \%, D. brevis/Total Demodex.

Univariate analysis indicated that severity of corneal changes was significantly correlated with sex, lid margin abnormalities, D. brevis \%, and meiboscore (all $\mathrm{P}<0.05)$ in the young group. However, the severity of corneal changes was not correlated with any of these factors except for meiboscore in the elderly group (Table 2). Multivariate ordinal logistic regression revealed that higher D. brevis $\%$, more lid margin abnormalities, and severe MGL were predictors of corneal changes severity in the young group (Table 2). However, severe MGL was a predictor of the presence of SPK in the elderly group (Table 2).

\section{Prevalence and potential risk factors of MGL}

Severe MGL was detected in 34 out of 91 (37.4\%) patients in the young group, which was higher than that in the elderly $(15.2 \%, \mathrm{P}<0.001$, Table 1$)$. In univariate analysis, the severity of MGL was correlated with female sex, D. brevis \%, TBUT, TMH, lid margin abnormalities, and eyelash disorder in the young group, but only age in the elderly group (Table 3). Multivariate ordinal logistic regression revealed that female sex, D. brevis \%, and severer lid margin abnormalities were significantly correlated with more severe MGL. However, age was the only predictor of MGL in the elderly group (Table 3).

\section{Representative case 1: young patient with ocular demodicosis}

A 12-year-old girl complained of redness and decreased vision in both eyes for two years. She was previously treated for herpes simplex keratitis and allergic conjunctivitis but failed to respond to antiviral and anti-allergy therapies. On examination, her visual acuity was 20/20 and 20/40, respectively. Lash sampling revealed four $D$. brevis and two $D$. folliculorum mites. The right eye had milder blepharitis with inferior corneal SPK (Figure 1A). The left eye had more blepharitis, especially in the upper lid in contact with the corneal epithelial defect associated with stromal infiltrate and neovascularisation (Figure 1B). Both eyes had a meiboscore of four (Figure 1C,D). 
Table 3 Probable risk factors of meibomian gland loss in young and elderly patients.

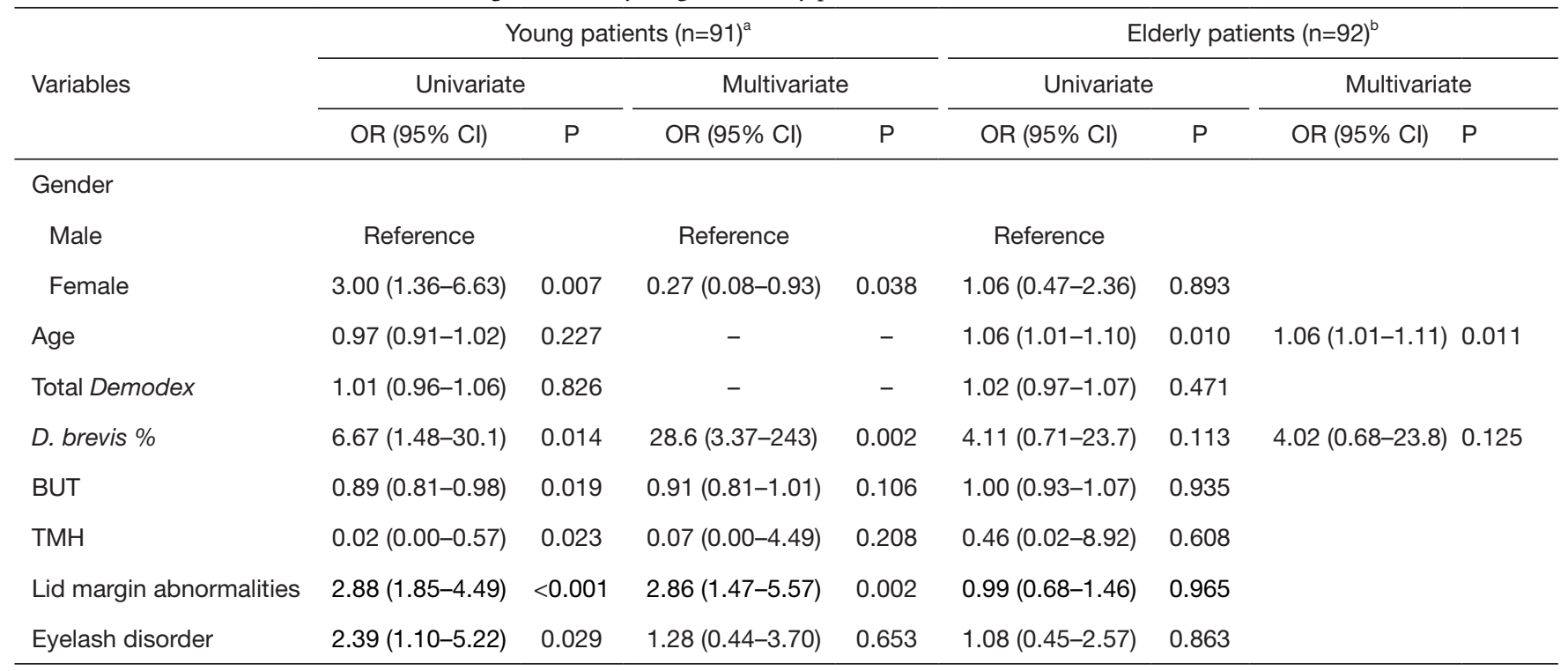

Variables which $\mathrm{P}<0.15$ were included in the multivariate ordinal logistic regression analysis. $\mathrm{Cl}$, confidence interval; OR, odds ratio; $\mathrm{BUT}$, tear break-up time; TMH, tear meniscus height. D. brevis, Demodex brevis; D. brevis \%, D. brevis/total Demodex.
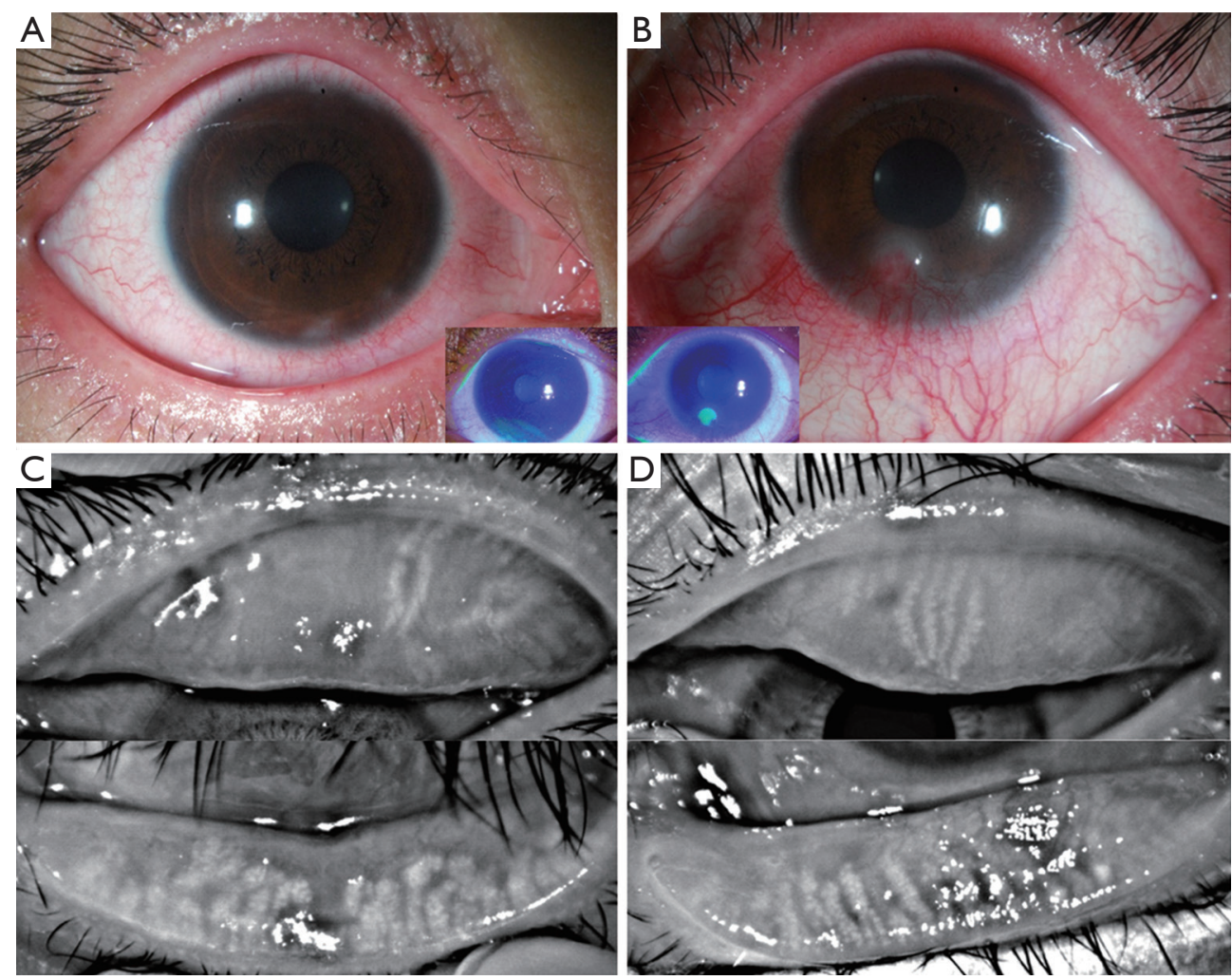

Figure 1 Representative case 1 . The left eye presented with grade-2 corneal change with an epithelium defect associated with stromal infiltration and vascularisation and lid/conjunctival inflammation (A,B). Blepharitis appeared to be worse in the upper lid and the neovascularisation grows into the cornea from below. Meibography showed that meibomian gland loss was severe in the upper lids (C,D). 

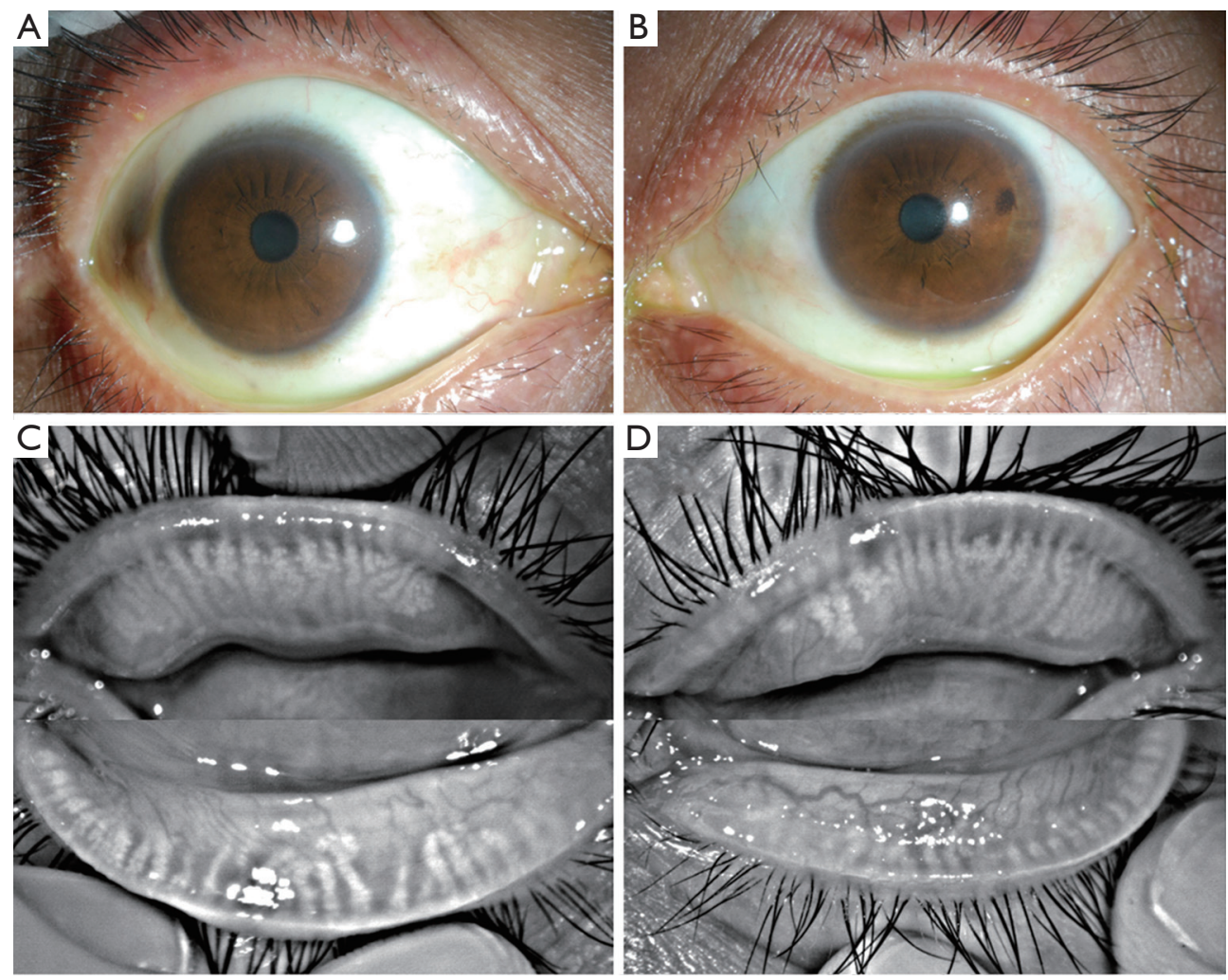

Figure 2 Representative case 2. Both eyes presented with meibomian gland orifice plugging, irregular lid margin, mild conjunctival hyperaemia and normal cornea of both eyes (A,B). Meibography showed that meibomian gland loss was mild in the upper and lower lids (C,D).

\section{Representative case 2: elderly patient with ocular demodicosis}

A 52-year-old woman complained of irritation and dryness for four years. She was previously diagnosed with MGD and was treated with artificial tears. On examination, her vision was $20 / 20$ and 20/25 respectively. Lash sampling detected nine $D$. folliculorum. Both eyes had meibomian gland orifice plugging, irregular lid margin, and mild conjunctival hyperaemia (Figure $2 A, B$ ). Meibography showed mild MGL in both eyes (Figure 2C,D).

\section{Discussion}

In this study, we found that $D$. brevis infestation was significantly higher and corneal changes and MGL were more severe in young patients with ocular demodicosis than in elderly patients. The diagnostic criteria for ocular demodicosis are still controversial. This study showed that Demodex profiles differ between young and elderly patients with ocular demodicosis. Therefore, age might be considered when setting diagnostic criteria for ocular demodicosis.

Our results showed that there was no significant difference in Demodex counts between young and elderly patients, which was inconsistent with previous studies showing that demodicosis is highly age-dependent $(8,13,26)$. We further discovered that the $D$. brevis counts and D. brevis $\%$ of the young group were higher than that of the elderly group. We speculate that this may be closely related to the parasitic environment of $D$. brevis and the more active meibum secretion in young individuals, which is beneficial to $D$. brevis parasitism $(2,27)$. It should be noted that the mean demodex count in our study was different from other published studies $(28,29)$. The plausible reasons include the different ages ranges and different numbers of epilated lashes in those studies.

It has been exhibited that Demodex plays an important role in blepharitis and ocular surface irritation (30-32). Herein, we noted that elderly patients with ocular demodicosis often complain of eye dryness, eye fatigue, and itching. On the contrary, blurred vision and eye pain are more common in young patients, perhaps because eye 
dryness and MGD are the main manifestations in elderly patients, and mainly cause ocular surface discomfort. However, in young patients, severe MGL, corneal changes, and even visual impairment are the dominant manifestations.

Severe MGL was found in young patients, and multiple logistic analyses illustrated that the severity of MGL was significantly correlated with $D$. brevis \%. Previous studies have indicated that ageing influences the structure and/ or function of the meibomian gland by decreasing the density of acinar units and acinar diameter in meibomian glands (33-36). In our study, elderly patients mainly showed MGD, but their MGL was classified as mild to moderate. Multivariable logistic regression analysis demonstrated that age was the only risk factor for MGL. Therefore, ageing, but not Demodex infestation itself, may be the major cause of MGL in elderly patients with ocular demodicosis.

Moreover, we observed that the prevalence of severe MGL was higher in the young group. Further multivariable logistic regression analysis indicated that lid margin abnormalities and D. brevis \% may be the main risk factors of MGL in young patients, suggesting that D. brevis may have a higher potential for triggering severe MGD in young patients. Further studies on the pathogenicity of D. brevis are warranted.

In the present study, it can be clearly seen that the young group had a higher prevalence of corneal changes involving the stroma, such as infiltration or ulceration, while SPK was the main corneal change in elderly people. Our previous research and the findings in this study provide strong support to D. brevis or D. brevis \% as a risk factor for corneal changes in young patients with ocular demodicosis (13-15). This study revealed that there are relatively fewer $D$. brevis mites and milder corneal changes in elderly patients, which further supports our notions that $D$. brevis plays a more important role in the pathogenesis of ocular demodicosis. The question of why young people with ocular demodicosis were detected with more $D$. brevis mites needs to be further studied.

Several limitations of our study should also be mentioned. Although lash sampling and microscopic examination are commonly applied to identify mites in lashes, it may miss Demodex mites that accumulate in the follicles of eyelashes. In vivo confocal microscopy may provide a more complete examination of follicles. The age groups were chosen relatively arbitrary. The participants enrolled in our study are patients who come to the cornea department. Their conditions might be more severe than those went to the general ophthalmology department or family doctors. In conclusion, ocular demodicosis has different clinical features between young and elderly patients, and the predominance of $D$. brevis may be the potential cause. This may be related to the fact that active secretion by the meibomian gland in young people could be conducive to the life of D. brevis. Therefore, the pathological mechanism of Demodex needs to be further explored.

\section{Acknowledgments}

Funding: This study is supported by grants (81770892, 82070922) from the National Natural Science Foundation of China, a grant (2019A1515012012) from the Science Foundation of Guangdong Province, and a grant (303020101) from High-level Hospital Foundation. The sponsors or funding organisations had no role in the design or conduct of this research; collection, management, analysis, and interpretation of the data; and preparation, review, or approval of the manuscript.

\section{Footnote}

Reporting Checklist: The authors have completed the STROBE reporting checklist. Available at http://dx.doi. org/10.21037/atm-20-7715

Data Sharing Statement: Available at http://dx.doi. org/10.21037/atm-20-7715

Peer Review File: Available at http://dx.doi.org/10.21037/ atm-20-7715

Conflicts of Interest: All authors have completed the ICMJE uniform disclosure form (available at http://dx.doi. org/10.21037/atm-20-7715). The authors have no conflicts of interest to declare.

Ethical Statement: The authors are accountable for all aspects of the work in ensuring that questions related to the accuracy or integrity of any part of the work are appropriately investigated and resolved. The study was approved by the Ethics Committee of Zhongshan Ophthalmic Center, Sun Yat-sen University (No. 2017KYPJ042), and complied with the Declaration of Helsinki (as revised in 2013) for human research. All participants were given a full explanation of the study, and written informed consent was obtained. 
Open Access Statement: This is an Open Access article distributed in accordance with the Creative Commons Attribution-NonCommercial-NoDerivs 4.0 International License (CC BY-NC-ND 4.0), which permits the noncommercial replication and distribution of the article with the strict proviso that no changes or edits are made and the original work is properly cited (including links to both the formal publication through the relevant DOI and the license). See: https://creativecommons.org/licenses/by-nc-nd/4.0/.

\section{References}

1. English FP, Nutting WB. Demodicosis of ophthalmic concern. Am J Ophthalmol 1981;91:362-72.

2. Liu J, Sheha H, Tseng SC. Pathogenic role of Demodex mites in blepharitis. Curr Opin Allergy Clin Immunol 2010;10:505-10.

3. Rufli T, Mumcuoglu Y. The hair follicle mites Demodex folliculorum and Demodex brevis: biology and medical importance. A review. Dermatologica 1981;162:1-11.

4. Lacey N, Kavanagh K, Tseng SC. Under the lash: Demodex mites in human diseases. Biochem (Lond) 2009;31:2-6.

5. Gao YY, Di Pascuale MA, Elizondo A, et al. Clinical treatment of ocular demodecosis by lid scrub with tea tree oil. Cornea 2007;26:136-43.

6. Zhang XB, Ding YH, He W. The association between demodex infestation and ocular surface manifestations in meibomian gland dysfunction. Int J Ophthalmol 2018;11:589-92.

7. Liang L, Liu Y, Ding X, et al. Significant correlation between meibomian gland dysfunction and keratitis in young patients with Demodex brevis infestation. $\mathrm{Br} \mathrm{J}$ Ophthalmol 2018;102:1098-102.

8. Liang L, Safran S, Gao Y, et al. Ocular demodicosis as a potential cause of pediatric blepharoconjunctivitis. Cornea 2010;29:1386-91.

9. Kheirkhah A, Casas V, Li W, et al. Corneal manifestations of ocular demodex infestation. Am J Ophthalmol 2007;143:743-9.

10. Fromstein SR, Harthan JS, Patel J, et al. Demodex blepharitis: clinical perspectives. Clin Optom (Auckl) 2018;10:57-63.

11. Czepita D, Kuzna-Grygiel W, Kosik-Bogacka D. Investigations on the occurrence as well as the role of Demodex follicuforum and Demodex brevis in the pathogensis of blepharitis. Klin Oczna 2005;107:80-2.

12. Norn MS. Incidence of Demodex folliculorum on skin of lids and nose. Acta Ophthalmol (Copenh) 1982;60:575-83.

13. Nowomiejska K, Lukasik P, Brzozowska A, et al.

Prevalence of Ocular Demodicosis and Ocular Surface Conditions in Patients Selected for Cataract Surgery. J Clin Med 2020;9:3069.

14. Zhang N, Liu Y, Wen K, et al. Prevalence of Ocular Demodex Infestation in Children: An Epidemiological Survey in South China. Eye Contact Lens 2021;47:60-4.

15. Luo X, Li J, Chen C, et al. Ocular Demodicosis as a Potential Cause of Ocular Surface Inflammation. Cornea 2017;36 Suppl 1:S9-S14.

16. Gao YY, Di Pascuale MA, Li W, et al. High prevalence of Demodex in eyelashes with cylindrical dandruff. Invest Ophthalmol Vis Sci 2005;46:3089-94.

17. Kheirkhah A, Blanco G, Casas V, et al. Fluorescein dye improves microscopic evaluation and counting of demodex in blepharitis with cylindrical dandruff. Cornea 2007;26:697-700.

18. Murphy O, V OD, Lloyd-Mckernan A. The Efficacy of Warm Compresses in the Treatment of Meibomian Gland Dysfunction and Demodex Folliculorum Blepharitis. Curr Eye Res 2020;45:563-75.

19. Ayyildiz T, Sezgin FM. The Effect of Ocular Demodex Colonization on Schirmer test and OSDI Scores in Newly Diagnosed Dry Eye Patients. Eye Contact Lens 2020;46 Suppl 1:S39-S41.

20. Cheng AM, Hwang J, Dermer H, et al. Prevalence of Ocular Demodicosis in an Older Population and Its Association With Symptoms and Signs of Dry Eye. Cornea 2020. [Epub ahead of print]. doi: 10.1097/ ICO.0000000000002542.

21. Liang LY, Li J, Liu Y. Diagnostic criteria of demodex blepharitis. Zhonghua Yan Ke Za Zhi 2017;53:648-52.

22. Tomlinson A, Bron AJ, Korb DR, et al. The international workshop on meibomian gland dysfunction: report of the diagnosis subcommittee. Invest Ophthalmol Vis Sci 2011;52:2006-49.

23. Arita R, Itoh K, Maeda S, et al. Proposed diagnostic criteria for obstructive meibomian gland dysfunction. Ophthalmology 2009;116:2058-63.e1.

24. Horwath-Winter J, Berghold A, Schmut O, et al. Evaluation of the clinical course of dry eye syndrome. Arch Ophthalmol 2003;121:1364-8.

25. Arita R, Itoh K, Inoue $\mathrm{K}$, et al. Noncontact infrared meibography to document age-related changes of the meibomian glands in a normal population. Ophthalmology 2008;115:911-5.

26. Post CF, Juhlin E. Demodex Folliculorum and Blepharitis. 
Arch Dermatol 1963;88:298-302.

27. Czepita D, Kuzna-Grygiel W, Czepita M, et al. Demodex folliculorum and Demodex brevis as a cause of chronic marginal blepharitis. Ann Acad Med Stetin 2007;53:63-7; discussion 7.

28. Mizuno M, Kawashima M, Uchino M, et al. DemodexMite Infestation in Cilia and its Association With Ocular Surface Parameters in Japanese Volunteers. Eye Contact Lens 2020;46:291-6.

29. Rabensteiner DF, Aminfar H, Boldin I, et al. Demodex Mite Infestation and its Associations with Tear Film and Ocular Surface Parameters in Patients with Ocular Discomfort. Am J Ophthalmol 2019;204:7-12.

30. Lee SH, Chun YS, Kim JH, et al. The relationship between demodex and ocular discomfort. Invest Ophthalmol Vis Sci 2010;51:2906-11.

31. Kabataş N, Dogan AS, Kabatas EU, et al. The Effect of Demodex Infestation on Blepharitis and the Ocular Symptoms. Eye Contact Lens 2017;43:64-7.

32. Murphy O, O'Dwyer V, Lloyd-McKernan A. Ocular Demodex folliculorum: prevalence and associated

Cite this article as: Li J, Luo X, Liao Y, Liang L. Age differences in ocular demodicosis: Demodex profiles and clinical manifestations. Ann Transl Med 2021;9(9):791. doi: 10.21037/ atm-20-7715 symptoms in an Irish population. Int Ophthalmol 2019;39:405-17.

33. Arita R, Mizoguchi T, Kawashima M, et al. Meibomian Gland Dysfunction and Dry Eye Are Similar but Different Based on a Population-Based Study: The Hirado-Takushima Study in Japan. Am J Ophthalmol 2019;207:410-8.

34. Wei A, Hong J, Sun X, et al. Evaluation of agerelated changes in human palpebral conjunctiva and meibomian glands by in vivo confocal microscopy. Cornea 2011;30:1007-12.

35. Fasanella V, Agnifili L, Mastropasqua R, et al. In Vivo Laser Scanning Confocal Microscopy of Human Meibomian Glands in Aging and Ocular Surface Diseases. Biomed Res Int 2016;2016:7432131.

36. Schaumberg DA, Nichols JJ, Papas EB, et al. The international workshop on meibomian gland dysfunction: report of the subcommittee on the epidemiology of, and associated risk factors for, MGD. Invest Ophthalmol Vis Sci 2011;52:1994-2005. 